\title{
Optical Environments of M51 X-ray Sources
}

\author{
Roy E. Kilgard ${ }^{1,3}$, Andrea H. Prestwich ${ }^{1}$, Martin J. Ward ${ }^{2}$ \\ and Timothy P. Roberts ${ }^{3}$ \\ ${ }^{1}$ Harvard-Smithsonian Center for Astrophysics, Cambridge, MA 02138, USA \\ email: rkilgard@cfa.harvard.edu \\ ${ }^{2}$ Department of Physics, University of Durham, Durham, DH1 3LE, UK \\ ${ }^{3}$ Department of Physics, University of Leicester, Leicester, LE1 7RH, UK
}

\begin{abstract}
The X-ray populations of spiral galaxies consist almost entirely of accreting X-ray binaries and supernova remnants. For the most luminous sources, it is possible to use X-ray spectroscopy and variability studies to gain insights into the nature of the sources. However, without unambiguously identified optical counterparts, it is impossible to definitively classify sources as, e.g. high-mass or low-mass X-ray binaries. The nearby interacting galaxy M51 is one of the best-studied galaxies across all wavelengths. At a distance of around $8 \mathrm{Mpc}$, it is possible to resolve features on scales of a few parsecs both in the X-ray and the optical. Recently, M51 was observed with the Advanced Camera for Surveys on the Hubble Space Telescope as part of a Hubble Legacy program. M51 has also been observed 3 times with the Chandra X-ray observatory. Combining these two datasets, we present initial results on the optical environments of M51 X-ray sources as the first part of a truly multi-wavelength study of X-ray sources in nearby galaxies.
\end{abstract}

Keywords. X-rays: galaxies, X-rays: binaries, galaxies: spiral.

\section{Introduction}

The discrete X-ray source populations of spiral galaxies consist almost entirely of accreting X-ray binaries and supernova remnants (Fabbiano (1989)). Prior to the launch of the Chandra X-ray Observatory in 1999, it was possible only to study these sources in detail in the most nearby galaxies: the Milky Way, the Magellanic Clouds, and, to a lesser extent, M31. However, with the large collecting area and sub-arcsecond spatial resolution of Chandra, it is possible to study X-ray source populations in galaxies well beyond $10 \mathrm{Mpc}$. This has led to much understanding about the relation between X-ray binary luminosity functions and star-formation (Kilgard et al. (2002), Grimm, Gilfanov, \& Sunyaev (2003)) and the relation between X-ray color and source classification (Prestwich et al. (2003)).

In order to study the X-ray source population of spiral galaxies in detail, a survey of 11 galaxies has been performed with Chandra, with detailed results presented in Kilgard et al. (2005). This work characterizes the X-ray population based entirely on the Xray spectral and temporal properties. However, it is impossible to truly understand the nature of an X-ray source without detecting an unambiguous counterpart in at least one other wavelength. As such, we have begun an extensive archival analysis project to collect as much multiwavelength data as possible for our sample of 11 galaxies, beginning with M51. The goals of the project are: to completely classify all X-ray sources in nearby galaxies; to determine the binary fraction within nearby galaxies and compare with the star formation rate and history as determined from observations at other wavelengths; to compare X-ray populations of different environments within individual galaxies, e.g. bulge vs. disk, arm vs. inter-arm, tidal tails, halos, etc. 
The nearby (8.4 Mpc, Feldmeier, Ciardullo \& Jacoby (1997)) interacting galaxy M51 has been studied extensively in almost all wavelengths. It is the closest example of an interacting galaxy pair, with massive star formation along the tidal tail and in the northern arm (Larsen (2000)). In early 2005, the Hubble Space Telescope observed M51 with the Advanced Camera for Surveys (ACS) in 3 wide optical filters and in a narrow $H \alpha$ filter (the F435W, F555W, F814W and F658N filters) as part of a Hubble Legacy program to celebrate the 15th anniversary of the telescope. The data was combined and cleaned at the STScI, producing science quality images of $8,600 \times 12,200$ pixels at 0.05 ” per pixel, and reaching limiting magnitudes of 27.3 , 26.5 and 25.8 in B, V and I, respectively. As such, the data provides an excellent counterpart to the high-resolution X-ray data provided by Chandra. M51 has been observed 3 times by Chandra for a total of around $100 \mathrm{ks}$. We detect around 120 discrete X-ray sources plus diffuse emission to a limiting luminosity of $3 \times 10^{36} \mathrm{erg} \mathrm{s}^{-1}$ in the $0.3-8 \mathrm{keV}$ band (results are presented in Terashima \& Wilson (2004) and Kilgard et al. (2005)).

\section{Data Analysis}

In order to correctly locate potential optical counterparts to the X-ray sources, it is necessary to register all the images to a common world coordinate system. We utilize the USNO B1.0 catalog, with astrometric accuracy of around 0.2" (Monet et al. (2003)). In performing this correction, it is necessary to select the catalog stars by hand, as an analysis of the ACS data shows that many of the objects identified as stars in the catalog are actually not discrete sources: some are more than one point source with very small separation, but many are extended galaxies. Once these have been eliminated, the coordinates of the corrected optical and X-ray images are good to around $0.4^{\prime \prime}$, slightly better than the Chandra ACIS pixel size of $0.5^{\prime \prime}$. At the distance of M51, this corresponds to an absolute accuracy of around 16 parsecs.

At the distance of M51, the limiting V magnitude of 26.5 corresponds to an absolute magnitude of $M_{v}=-3.1$, which allows us to directly detect most $\mathrm{O}$ and $\mathrm{B}$ stars. In addition, the astrometric accuracy will allow us to place sources within individual star clusters or HII regions. Of the 116 X-ray sources in M51, we exclude 16 sources: 5 because they are not covered by the ACS field-of-view, and 11 that are found in the extremely dusty nuclear region of M51a.

\section{First Results}

We shall consider source coincidences of 4 types: apparently unambiguous matches, matches with star clusters, matches with $H \alpha$ structures, and X-ray sources with no optical matches.

- X-ray sources with unambiguous matches: Of the $100 \mathrm{X}$-ray sources considered, 25 have discrete optical counterparts: 4 are background AGN, 3 are background galaxies, 1 is a foreground star, and one is the nucleus of M51b. The remaining 16 have stellar counterparts that appear to be associated with M51. 11 appear to be O or B type stars, while the remaining 5 are in heavily obscured dust lanes where it is hard to obtain accurate photometry. All 16 of the sources have X-ray colors that are consistent with the sources being soft X-ray binaries, likely HMXBs in the high/soft state.

- $X$-ray sources in star clusters: 9 of the sources are coincident with young star clusters. Of these, 4 are extremely faint in X-ray, one has extremely soft X-ray color (either a SNR or supersoft source), and 4 have colors consistent with X-ray binaries. Of the 4 

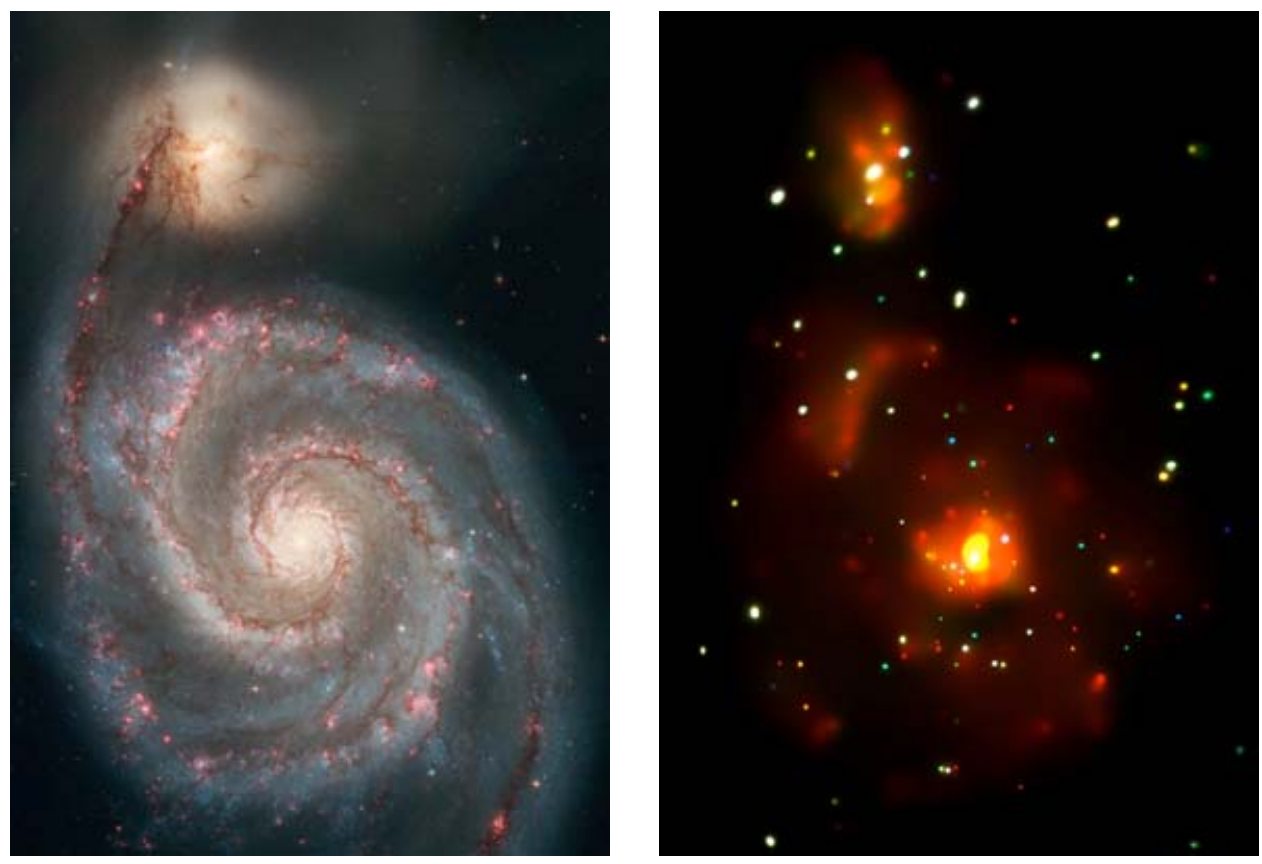

Figure 1. Left: The combined optical image of M51 red is $H \alpha$, green is $\mathrm{V}$ band and blue is $\mathrm{B}$ band. Right: The adaptively smoothed, combined X-ray image; red is $0.3-1 \mathrm{keV}$, green is $1-2 \mathrm{keV}$, and blue is $2-8 \mathrm{keV}$.

X-ray binary candidates, 3 are ultraluminous X-ray sources, with luminosities in excess of $10^{39} \mathrm{erg} \mathrm{s}^{-1}$.

- $X$-ray sources and $H \alpha$ features: A large number of sources $(24 / 100)$ is coincident with features in the $H \alpha$. Most of these features are either shell-like or circular, and a few pixels across in the optical, corresponding to sizes of a few parsecs (each pixel in the optical images is around 2 parsecs across). The X-ray colors are consistently soft: 9 have SNR colors, 3 have colors that fall on the SNR/XRB cusp, and 9 have too few counts to classify, but appear to have soft colors. Only 3 have colors typical of X-ray binaries, and it is worth noting that, as one adds absorption, the colors of sources in the SNR category will move into the XRB category in the color classification scheme used (Kilgard et al. (2005)). 7 of the 24 sources are also coincident with $20 \mathrm{~cm}$ sources (L. Maddox, private communication), thus suggesting that X-ray observations may be sensitive to SNR of different ages.

Amongst these sources, there appears a clear divide in luminosity space. 7 of the sources have luminosities greater than $6 \times 10^{37} \mathrm{erg} \mathrm{s}^{-1}$ : the $3 \mathrm{SNR} / \mathrm{XRB}$ cusp sources, the 3 XRB-candidates, and one source with SNR colors. This source is a ULX with a cool disk blackbody spectrum and is a good candidate for an intermediate-mass black hole (Miller, Fabian, \& Miller (2004)).

- No optical matches: Of the remaining sources, 19 have more than one O or B star in the X-ray error ellipse. The remaining sources are largely located within dust lanes, making it difficult to detect optical counterparts. The X-ray colors of these sources are typical of harder X-ray binaries: either LMXBs or HMXBs with heavy absorption. We also expect about 5-10 of these sources to be due to background contamination. 


\section{Conclusions}

In an analysis of X-ray and optical data of M51, it is possible to classify around half of the X-ray source population as either HMXBs or SNR, using a combination of the X-ray colors of the sources and coincidence with optical star clusters, individual stars, or extended features in the $H \alpha$ that appear to be SNR. We can also remove a substantial portion of the foreground and background contamination by identifying direct optical counterparts to the sources. In addition, for those sources with no detected optical counterpart, we are able to make inferences as to their classification. For example, the absence of optical counterparts to any M51b X-ray sources except the nucleus indicates that the population is dominated by LMXBs. With this combination of X-ray color analysis and identification of optical counterparts, it is now possible to study separately the HMXB, LMXB, and SNR populations of spiral galaxies beyond the Milky Way.

\section{Acknowledgements}

Based on observations made with the NASA/ESA Hubble Space Telescope, obtained via the Hubble Legacy Program, at the Space Telescope Science Institute, which is operated by the Association of Universities for Research in Astronomy, Inc., under NASA contract NAS 5-26555. These observations are associated with program HST-AR-10669.01. A. Prestwich would also like to acknowledge support from the Chandra X-ray Center, operated under NASA contract NAS 8-39073.

\section{References}

Fabbiano, G. 1989, ARA\&A 27, 87

Feldmeier, J.J., Ciardullo, R., \& Jacoby, G.H. 1997, ApJ 479, 231

Grimm, H.-J., Gifanov, M., \& Sunyaev, R. 2003, MNRAS 339, 793

Kilgard, R.E., Kaaret, P., Krauss, M.I., Prestwich, A.H., Raley, M.T., \& Zezas, A. 2002, ApJ 573,138

Kilgard, R.E., Cowan, J.J., Garcia, M.R., Kaaret, P., Krauss, M.I., McDowell, J.C., Prestwich, A.H., Primini, F.A., Stockdale, C.J., Trinchieri, G., Ward., M.J., \& Zezas, A. 2005, ApJS 159,214

Larsen, S.S. 2000, MNRAS 319, 893

Miller, J.M., Fabian, A.C., \& Miller, M.C. 2004, ApJ, 614, 117

Monet, D.G., Levine, S.E., Canzian, B., Ables, H.D., Bird, A.R., Dahn, C.C., Guetter, H.H., Harris, H.C., et al. 2003, AJ 125, 984

Prestwich, A.H., Irwin, J.A., Kilgard, R.E., Krauss, M.I., Zezas, A., Primini, F., Kaaret, P., \& Boroson, B. 2003, ApJ 595, 719

Terashima, Y. \& Wilson., A.S. 2004, ApJ 601, 735

\section{Discussion}

GARCIA: Tell us more about the ULX/IMBH candidate.

KILGARD: It is a fuzzy blob on $\mathrm{H} \alpha$, and detected on $\mathrm{B}$ and $\mathrm{V}$.

Di Stefano: Are any of the ULXs coincident with features in the $H \alpha$ ?

KILGARD: Yes, one of the ULXs is coincident with a shell-like feature in the $H \alpha$. It has a soft spectrum coincident with being an X-ray binary in the high-soft state. 